\title{
Single or double-layer uterine closure techniques following cesarean: An ongoing debate
}

\author{
Şafak Yılmaz Baran${ }^{1}$, Hakan Kalaycı², Gülşen Doğan Durdağ ${ }^{2}$, Selçuk Yetkinel², Songül \\ Alemdaroğlu², Tayfun Çok $^{2}$, and Esra Bulgan Kılıçdağ ${ }^{2}$ \\ ${ }^{1}$ Affiliation not available \\ ${ }^{2}$ Baskent Universitesi Adana Uygulama ve Arastirma Merkezi
}

May 11, 2020

\begin{abstract}
Objectives To examine the effects of single- and double-layer uterine closure techniques on uterine scar healing following cesarean delivery. Design Randomised controlled trial Setting University Hospital of Başkent from July 2018 to September 2019 Population This study assessed a total of 282 women between 18-45 years of age who had singleton pregnancies and had not previously undergone uterine surgeries. Methods Participants were randomized into two following treatment groups: singlelayer closure with locking and double-layer closure with locking in the first layer, but not in the second layer (NCT03629028). Participants were evaluated at 6-9 months after cesarean section by saline infusion sonohysterography to assess cesarean delivery scar defects. Experienced sonographers who were not informed about the uterine closure technique conducted these procedures. Results Of the 225 final participants, 116 received the double-layer closure technique, while 109 received the single-layer technique. In this regard, the niche rates and median niche depths based on transvaginal ultrasounds and sonohysterography investigations were $21 \%, 0.9 \pm 1.8 \mathrm{~mm}$, and $41 \%, 2.1 \pm 1.9 \mathrm{~mm}(\mathrm{p}<0.001, \mathrm{p}<0.001)$, respectively. The niche rates were $37 \%$ for the single-layer group and $45.7 \%$ for the double-layer group $(\mathrm{p}=0.22)$. Median niche widths were higher in the double-layer group $(\mathrm{p}=0.006)$. Conclusions The single- and double-layer closure techniques did not produce different impacts on uterine scare niche development. However, the median niche width was higher for the double-layer closure group. Tweetable abstract There was no difference between single- and double layer closure in the rate of scar niche development following cesarean delivery. Keywords cesarean delivery, isthmocele, niche, uterine closure
\end{abstract}

\section{INTRODUCTION}

Increasing rates of cesarean delivery $(\mathrm{CD})$ in the world mean that a growing number of women are experiencing related complications. ${ }^{1}$ While short-term problems associated with the procedure include bleeding, and infection, there are also serious long-term issues, such as placenta adhesion anomalies, CD scar defects, uterine rupture, dehiscence, and caesarean delivery scar pregnancies. Further, many patients complain of postmenstrual spotting, dysmenorrhea, and pelvic pain, which may also be associated with these complications. ${ }^{2,3}$

There is no clear consensus on the best uterine closure technique for preventing cesarean scar defects. However, it is known that both the surgical suture technique and mechanical stresses affecting the surgical scar are the most important factors related to incision integrity. ${ }^{4}$ Several different techniques are used to close the uterus after cesarean, including single- and double-layer closures with/without locking and either passing through or avoiding the decidua. A variety of different suture materials are also available. Here, the main focus is on the uterine closure technique, especially for minimizing postoperative uterus rupture/dehiscence and caesarean delivery scar defects. Further, these techniques are modifiable parameters, with many recent studies having attempted to determine a standard. ${ }^{5}$ Nevertheless, no such standard has been established. For instance, while the Misgav Ladach single-layer continuous uterine closure with locking is prevalent 
throughout the world, ${ }^{6}$ studies have reported different results. ${ }^{7-10}$ A meta-analysis also showed a fourfold risk of uterine rupture in future pregnancies among patients whose uteri were closed using a single-layer locking technique when compared to those who were treated with a double-layer technique. ${ }^{11}$ Further, Stegwee et al. (2019) found that patients whose uterine incisions were closed by double-layer following cesarean section (CS) experienced greater advantages in terms of residual myometrium thickness (RMT), healing ratio (residual myometrium thickness/adjacent myometrium thickness), and dysmenorrhea. ${ }^{2}$ However, another meta-analysis conducted by Di Spiezio Sardo et al. (2017) found no significant differences between singleand double-layer closures in terms of niche development, uterine dehiscence, or rupture. ${ }^{10}$ As such, there is no current consensus about the specific uterine closure technique that best minimizes the risk of uterine rupture and/or caesarean delivery scar defect. This study therefore investigated the effects of single- and double-layer closures of the uterus in regard to niche development and residual myometrium thickness at 6-9 months after CS.

\section{MATERIALS AND METHODS}

\section{Design and Participants}

This study assessed CD patients who were admitted to the Obstetrics and Gynecology Clinic of the Başkent University Adana Dr. Turgut Noyan Research and Application Center between July 2018 and September 2019. Specifically, participants were singleton pregnancy patients between 18-45 years of age who were in gestational weeks 24-41 and had not previously received any uterine operations such as CS or myomectomy. Patients with multiple pregnancies and/or chronic inflammatory diseases such as systemic lupus erythematous, rheumatoid arthritis, and insulin-dependent diabetes mellitus were excluded. All patients who met the inclusion criteria were randomized into two treatment groups (i.e., single- or double-layer closures).

Patients who agreed to participate in this study were informed about the purpose prior to their operations and each provided signed written consent.

Participants were also assessed for maternal age, gravidity, parity, body mass index, smoking status, diabetes mellitus, hypertensive diseases of pregnancy, and medical history. Regardless of whether they had contractions before surgery, this study also determined patient CD indications, levels of cervical dilatation, and how CD was established (i.e., elective or emergent).

\section{Randomization and Masking}

A table of random numbers was used as simple randomization method for placing participants into the two treatment groups. The Research Randomizer website (https://www.randomizer.org/) was used to create this table. Before the intervention, the next envelope among a previously prepared, sealed, and number-ordered stack was opened by a nurse for each procedure.

\section{Surgery Techniques}

Six operators performed the CD operations. Access to the abdomen was achieved via Pfannenstiel incision, while the Kerr incision technique was applied to the uterus for all patients. Patients in the single-layer group received uterus closures with locking, while patients in the double-layer group received uterus closures with locking in the first layer, but without locking and using a parallel Lembert-type imbricating stitch in the second layer. The decidua was deliberately excluded during all suturations. A synthetic absorbable suture material (Vicryl 1.0, Ethicon, Somerville, NJ, USA) was used during all uterus closures, with corner stitches applied to all patients. Additional suture usages and numbers were reported only in cases of bleeding. Both uterine closure and whole operation times were recorded by the respective operating room technicians. Finally, intravenous prophylactic antibiotics (2 g cefazolin sodium) and uterotonic medications (10 IU oxytocin) were routinely applied during all CD procedures.

\section{Follow-Up}

Postoperative follow-ups were completed in March 2020. All participants were called after delivery and invited to complete sixth-month evaluations. However, participants who experienced post-CD pregnancies, 
or uterine surgeries were excluded. All remaining participants were examined at six to nine months period after $\mathrm{CD}$ for niche presence in the scar region, niche measurements, scar shape, distance between niche and the external cervical os and residual myometrium thickness (RMT), and adjacent myometrium thicknesses (AMT). The distance between the niche and external os should be measured parallel to the top of the main niche, from the most distal niche point to external os. These examinations were conducted by two experienced sonographers who were blinded to clinical information regarding the closure technique. Procedures were specifically completed using a 4-10 MHz transducer (E8C-RS micro convex endocavity probe, Voluson S6, GE, Milwaukee, US), ultrasonography, and through the saline infusion sonohysterography (SIS) method. The cervix was first cleaned with povidone iodine while the patient was on the gynecological examination table prior to ultrasonography. The SIS process then progressed toward the inside of the endometrial cavity by passing from the cervical os with an intrauterine insemination cannula (intrauterine insemination catheter, Wallace Artificial Insemination Catheter, Smiths Medical International Ltd., Ashford, Kent, UK). A sterile saline solution was the applied to sufficiently distend the cavity, while ultrasonography was conducted via transvaginal probe. At this time, the presence of a hypoechogenic area with a depth of $2 \mathrm{~mm}$ or deeper in the CD scar region within the endometrial cavity was accepted as a niche. Niche dimensions were then determined via 3-axis measurements for depth, length, and width. Niche shape, distance to the external cervical os, RMT, and AMT were then evaluated. Figure 2 and 3 Figure 2 demonstrated saline infusion sonohysterography evaluation of patient's cesarean scar defects (length of niche, depth of niche, RMT, AMT, distance between niche and external os and width of niche).

During these follow-ups, patients were also asked about their menstruation processes and any experiences of cervical pain, menstruation pain, postmenstrual bleeding (PMB) in the form of spotting, and other abnormal bleeding patterns. Those who experienced bleeding for two days or more in the form of spotting after menstruation were defined as PMB. Participants who used intrauterine devices after CD and/or those who could not tolerate speculum application were examined via transvaginal ultrasonography (TV USG).

\section{Outcomes}

Primary outcomes were considered niche presence and measurements, while secondary outcomes were considered RMT, AMT, healing ratio, postmenstrual spotting, and dysmenorrhea.

\section{Statistical Analyses}

The sample size was calculated based on a study by Di Spiezio Sardo et al. (2017) titled Risk of Cesarean Scar Defect Following Single- vs Double-Layer Uterine Closure: Systematic Review and Meta-Analysis of Randomized Controlled Trials. ${ }^{10}$ Their study revealed niche formation rates for single- and double-layer closure types of $25 \%$ and $43 \%$, respectively. Based on tools available at the Power and Sample Size website (http://powerandsamplesize.com/Calculators), needed sample size was calculated at 141 for each treatment group with $90 \%$ power and 0.05 alpha error.

All data were statistically analyzed using the IBM SPSS 21.0 software. Categorical measurements were presented as frequencies and percentages, while continuous measurements were summarized as means and standard deviations (median and range when needed). Distributions were checked and the student's t-test was used for variables meeting the parametric test assumptions when comparing the continuous measurements based on groups, while the Mann Whitney U test was used for those that did not meet the parametric test assumptions. Finally, chi-squared or Fisher's test statistics were used to compare categorical variables. Statistical significance was set to 0.05 for all tests.

\section{RESULTS}

A total of 282 patients met the initial inclusion criteria for this study. Of these, 57 were excluded (20.2\%); 49 were lost to follow-up (17.4\%), six became pregnant (2.1\%), and two could not endure ultrasonography due to intolerances to pelvic examinations $(0.71 \%)$. Thus, 225 patients were fully evaluated. Niche assessment was evaluated via SIS in 221 patients, while TV USG was used for four patients due to the use of intrauterine devices or intolerance to SIS. Of the 225 total remaining participants, 109 were placed into the single-layer 
treatment group, while 116 were placed into the double-layer treatment group (Figure 1). Further, 165 (73.3\%) patients reported that menstruation had begun when they returned for checkups 6-9 months after CS.

Participants of both treatment groups were compared based on their maternal and obstetric characteristics as well as those related to CD operations. Except for shorter uterine closures and operation durations for single-layer closures, all factors were similar between groups (Table 1).

The CD indications were fetal presentation anomalies $(\mathrm{n}=36,16 \%)$, fetal distress $(\mathrm{n}=25,11.1 \%)$, discontinued labor $(\mathrm{n}=31,13.8 \%)$, maternal request $(\mathrm{n}=57,25.3 \%)$, cephalo-pelvic disproportion $(\mathrm{n}=47,20.9 \%)$, and reasons such as severe preeclampsia, maternal factors, fetal anomalies, and placenta anomalies $(\mathrm{n}=29,12.9 \%)$.

This study found that niche rates and mean depths for the TV USG and SIS patients were $21 \%, 0.9 \pm 1.8 \mathrm{~mm}$ and $41 \%, 2.1 \pm 1.9 \mathrm{~mm}$, respectively $(\mathrm{p}<0.001, \mathrm{p}<0.001)$. Niche positivity was determined for 93 patients, while more frequent $\mathrm{PMB}$ and dysmenorrhea were observed among patients for whom niches were determined ( $32.1 \%$ vs $5.2 \% ; \mathrm{p}<0.001,12.9 \%$ vs $4.6 \% ; \mathrm{p}=0.043)$. Niche shapes were triangular $(82 \%)$, oval $(10.1 \%)$, circular $(4.5 \%)$, square $(2.2 \%)$, and totally defective $(\% 1.1)$, while niche rates were $37 \%$ for the single-layer group and $45.7 \%$ for the double-layer group $(\mathrm{p}=0.22)$. No significant differences were found between the niche depth measurements used to define niche presence $(\mathrm{p}=0.10)$, nor were any significant differences found in niche width measurements $(\mathrm{p}=0.07)$. However, niche width was higher in the transvers plane among participants of the double-layer group when compared to those in the single-layer group $(\mathrm{p}=0.006)$ (Table 2). On the other hand, no significant intergroup differences were found in terms of RMT, AMT, healing ratio values, and PMB / dysmenorrhea symptoms (Table 2). Mean uterine closures and operation durations were shorter among the single-layer group $(\mathrm{p}<0.001, \mathrm{p}=0.001)$.

\section{DISCUSSION}

\section{Main Findings}

This study found no significant differences between single-layer (locked) and double-layer (locked in the first layer, unlocked in the second) uterus closures following CD in terms of niche development, median depth, niche length, RMT, AMT, or PMB. However median niche width was higher among participants of the double-layer closure group. The CD technique entails several variations designed to shorten operation duration, simplify the process, increase efficiency, reduce costs, reduce the risk of side effects, and lower postoperative morbidity levels and hospitalization times. ${ }^{12}$ This study observed similar niche rates among the single-layer closure group via easier techniques that took less time.

\section{Strengths and Limitations}

This study also had some limitations. First, some patients were lost to follow-up. Second, its single-center structure and provision of limited information on abnormal uterine bleeding symptoms prevented the longterm assessment of symptoms such as PMB. However, there were several strengths, including the implementation of a randomized controlled design, the use of one tertiary center entailing the same surgical techniques, the stipulation that all patients were undergoing their first CD, the double-blinded assessment procedure in which neither the physicians nor patients had knowledge about whether single- or double-layer closures were used, the examination of patients via SIS by two experienced sonographers, and the practice of waiting at least six months before conducting patient follow-ups, thus allowing sufficient tissue healing. Further, the sample size was calculated via power analysis based on niche and with a broad number of patients with $90 \%$ power. In addition, previous studies assessed CD scar defects only during the first three postoperative months or via transabdominal/TV USG, while many only presented RMT or niche results. As such, this study took niche and myometrial thickness measurements with multiple planes after at least six months based on the European Society for Gynaecological Endoscopy (ESGE) guidelines. Finally, previous studies accepted niche presence beginning at depths of $1 \mathrm{~mm}$, while this study required at least $2 \mathrm{~mm}$, thereby producing more specific results.

\section{Interpretation}


Passing through decidua during suturing was deliberately avoided in all operations performed in the context of this study. A previous study by Roberge et al. (2011) found that the locking closure technique which passes through the decidua produced better results in terms of tissue combination and healing. ${ }^{11}$ However, niche development was still possible. Additionally, it is not always possible to completely avoid crossing the decidua during such operations. It was thus emphasized that including the decidua in measurements less than $5 \mathrm{~mm}$ was acceptable, while crossing the decidua with full thickness leads to fusion and may increase niche development. ${ }^{4,13}$

A previous randomized controlled trial compared three different uterine closure techniques (locked singlelayer including the decidua, double-layer with locked first layer including the decidua, and double-layer with unlocked first layer excluding the decidua) in terms of RMT; while no differences were found between the single- and double-layer closure techniques with locked first layers, double-layer closures without locking resulted in thicker RMT when compared to locked single-layer closures. ${ }^{14}$ These findings supported a hypothesis suggested by Jelsema et al. (1993) in which the locking suture technique was thought to develop ischemic necrosis in tissues due to increased pressure. ${ }^{15}$ However, many surgeons prefer sutures with locking because they provide good hemostasis. Participants in this study who received double-layer closures also received first-layer locking. For that reason, there were no specific findings about locking effects. While more pronounced niches were observed in participants with double-layer closures, these differences were not statistically significant. This may be caused by the combination of second-layer closures with first-layer locking may have increased tissue stress and disrupted vascularization, the idea that this was caused by differences in technique cannot currently be proven.

On the other hand, the 3-plane niche measurements revealed that participants who received double-layer closures had significantly higher niche widths. However, it is difficult to measure niche width in the transverse plane in retrovert uteri. As no other published study has compared transverse plane measurements in this regard, this finding cannot be thoroughly discussed.

The current literature shows that SIS is a more reliable method of assessing cesarean delivery scar defects than TV USG. ${ }^{16,17}$ In this study, the rates of niche determination were $21 \%$ in TV USG and $41 \%$ in SIS. This makes it clear that SIS provides more accurate results. While the PMB rate was $32.1 \%$ in cases where isthmocele was detected, the rate was $5.2 \%$ among those without. Further, a previous broad-scoped study revealed more noticeable PMB complaints among patients in whom isthmocele was determined at least six months after CD when compared to others. ${ }^{18}$ The lower than expected PMB rates found in this study may be because some patients $(60 ; 26.7 \%)$ had not yet begun to menstruate. As such, more accurate results can be produced by assessing patients for PMB as much as one year after the procedure.

While a previous study that randomly divided patients into three groups (single-layer closure of the uterus without locking, single-layer closure with locking, and double-layer closure) found no significant intergroup differences in terms of niche development and RMT, a trend was found in which thicker RMT was produced via double-layer closures; however, as opposed to the $2 \mathrm{~mm}$ depths considered in this study, niche presence was accepted at $1 \mathrm{~mm}$ and deeper, while participants with multiple pregnancies and repeat CDs were included and all niche assessments were conducted via TV USG. ${ }^{9}$

A previous study by Tekiner et al. (2018) found no significant differences between single- and double-layer uterine closures based on niche assessments at the third postoperative month. ${ }^{19}$ However, their study was limited in that the double-layer group had higher rates of emergency CD, in which there is a tendency for niches to develop at an increased rate. ${ }^{19}$ As emergency cases were similarly distributed between groups in this study, it may be seen that similar results were produced.

While Di Spiezio Sardo et al. (2017) compared single- and double-layer uterine closures between participants of nine randomized controlled trials and found no intergroup differences in terms of isthmocele, RMT was thinner among single-layer closure patients. However, different uterine closure techniques were used between studies, which also implemented different niche definitions and measurement methods. For that reason, overall results were of the low-moderate evidentiary level. This study also found no significant intergroup 
differences in terms of RMT. ${ }^{10}$

A multi-centric study with a protocol that was published in 2019 divided a total of 2,290 patients into singleand double-layer closure groups. Symptoms were then assessed at the third month via TV USG/SIS, while surveys were conducted to provide long-term data. Here, single-layer closures were made without locking and without regards to crossing the decidua, while double-layer closures were made by passing through the endometrium, without locking in the first layer, and continuously without locking in the second layer. Preliminary results show that niche presence was significantly lower among participants with single-layer closures (79.4\% vs $83 \%$ ), but no differences were found in terms of PMB. ${ }^{20}$ Although the differences were not statistically significant, this study found similar niche rates among the single-layer closure group (37.7\% vs $\% 45.7 \% ; \mathrm{p}=0.22$ ). However, this issue must be clarified through future prospective studies that include greater numbers of participants.

\section{Conclusions}

No significant differences were found between participants who received single- and double-layer uterine closures following $\mathrm{CD}$ in terms of niche presence, median depth, or niche width, nor were any such differences found based on RMT/AMT or PMB. However, median niche width was significantly higher among participants of the double-layer group.

Future research is thus needed to evaluate the clinical importance of median niche width.

\section{Disclosure of interests}

The authors report relevant financial, personal, political, intellectual or religious conflicts of interests.

\section{Contribution to authorship}

Principal investigator: ŞYB

Concept and design: ŞYB, HK and TÇ

Data acquisition: ŞYB, HK, SA, GDD, SY and TÇ

Analysis of data: ŞYB, TÇ and EBK

Drafting of manuscript: ŞYB, TÇ

Review of manuscript: All authors

\section{Details of ethics approval}

Başkent University Institutional Review Board and Ethics Committee approved this study (Project No= KA18/71, approval date $=27$ July 2018). This research was registered by clinicaltrial.gov (NCT03629028), https://clinicaltrials.gov/ct2/show/NCT03629028.

\section{Funding}

This research was supported by University Research Fund.

\section{Acknowledgements}

We thank to Caner Baran M.D for valuable comments to improve our study and Oya Çok M.D. Prof for revising the final version of the article.

\section{References}

1. Bamberg C, Hinkson L, Henrich W. Cesarean scar niche and uterotomy closure technique. Acta Obstetricia et Gynecologica Scandinavica.2018;97(5):630-630. 
2. Stegwee SI, Jordans IPM, van der Voet LF, et al. Uterine caesarean closure techniques affect ultrasound findings and maternal outcomes: a systematic review and meta-analysis. BJOG: An International Journal of Obstetrics \&3 Gynaecology. 2018;125(9):1097-1108.

3. Kremer TG, Ghiorzi IB, Dibi RP. Isthmocele: an overview of diagnosis and treatment. Rev. Assoc. Med. Bras. 2019;65(5):714-721.

4. Vervoort AJMW, Uittenbogaard LB, Hehenkamp WJK, Brölmann HAM, Mol BWJ, Huirne JAF. Why do niches develop in Caesarean uterine scars? Hypotheses on the aetiology of niche development. Hum. Reprod.2015:dev240.

5. Caesarean section surgical techniques: a randomised factorial trial (CAESAR)*. BJOG: An International Journal of Obstetrics 83 Gynaecology. 2010;117(11):1366-1376.

6. Holmgren G, Sjoholm L, Stark M. The Misgav Ladach method for cesarean section: method description. Acta Obstet. Gynecol. Scand. Aug 1999;78(7):615-621.

7. Caesarean section surgical techniques: 3 year follow-up of the CORONIS fractional, factorial, unmasked, randomised controlled trial. The Lancet. 2016;388(10039):62-72.

8. Bamberg C, Dudenhausen J, Bujak V, et al. A Prospective Randomized Clinical Trial of Single vs. Double Layer Closure of Hysterotomy at the Time of Cesarean Delivery: The Effect on Uterine Scar Thickness. Ultraschall in der Medizin - European Journal of Ultrasound.2016;39(03):343-351.

9. Bamberg C, Hinkson L, Dudenhausen JW, Bujak V, Kalache KD, Henrich W. Longitudinal transvaginal ultrasound evaluation of cesarean scar niche incidence and depth in the first two years after single- or double-layer uterotomy closure: a randomized controlled trial. Acta Obstetricia et Gynecologica Scandinavica. 2017;96(12):1484-1489.

10. Di Spiezio Sardo A, Saccone G, McCurdy R, Bujold E, Bifulco G, Berghella V. Risk of Cesarean scar defect following single- vs

double-layer uterine closure: systematic review and meta-analysis of randomized controlled trials. Ultrasound in Obstetrics \& Gynecology. 2017;50(5):578-583.

11. Roberge S, Chaillet N, Boutin A, et al. Single- versus double-layer closure of the hysterotomy incision during cesarean delivery and risk of uterine rupture. International Journal of Gynecology $\&$ Obstetrics. 2011;115(1):5-10.

12. Turan C, Büyükbayrak EE, Onan Yilmaz A, Karageyim Karsidag Y, Pirimoglu M. Purse-string doublelayer closure: A novel technique for repairing the uterine incision during cesarean section. Journal of Obstetrics and Gynaecology Research. 2015;41(4):565-574.

13. Sholapurkar SL. Etiology of Cesarean Uterine Scar Defect (Niche): Detailed Critical Analysis of Hypotheses and Prevention Strategies and Peritoneal Closure Debate. J. Clin. Med. Res. 2018;10(3):166-173.

14. Roberge S, Demers S, Girard M, et al. Impact of uterine closure on residual myometrial thickness after cesarean: a randomized controlled trial. Am. J. Obstet. Gynecol. 2016;214(4):507.e501-507.e506.

15. Jelsema RD, Wittingen JA, Vander Kolk KJ. Continuous, nonlocking, single-layer repair of the low transverse uterine incision. J. Reprod. Med. May 1993;38(5):393-396.

16. Antila-Långsjö R, Mäenpää JU, Huhtala H, Tomás E, Staff S. Comparison of transvaginal ultrasound and saline contrast sonohysterography in evaluation of cesarean scar defect: a prospective cohort study. Acta Obstetricia et Gynecologica Scandinavica.2018;97(9):1130-1136.

17. Vikhareva Osser O, Jokubkiene L, Valentin L. Cesarean section scar defects: agreement between transvaginal sonographic findings with and without saline contrast enhancement. Ultrasound in Obstetrics and Gynecology. 2010;35(1):75-83. 
18. Antila RM, Mäenpää JU, Huhtala HS, Tomás EI, Staff SM. Association of cesarean scar defect with abnormal uterine bleeding: The results of a prospective study. European Journal of Obstetrics $\&$ Gynecology and Reproductive Biology. 2020;244:134-140.

19. Tekiner NB, Çetin BA, Türkgeldi LS, Yılmaz G, Polat İ, Gedikbaşı A. Evaluation of cesarean scar after single- and double-layer hysterotomy closure: a prospective cross-sectional study. Arch. Gynecol. Obstet. 2018;297(5):1137-1143.

20. Stegwee SI JI, van der Voet LF, Bongers MY, de Groot CJM, Lambalk CB, et al. . The effect of singleversus double-layer closure of the uterine caesarean scar on postmenstrual spotting: a multicentre, doubleblind, randomised controlled trial. Fact, Views $\&$ Vision. Journal of the European Society for Gynaecological Endoscopy. 2019;11 (Supplement 1).

\section{Table legends}

Table 1. Maternal and perinatal characteristics of patients in each study group

Table 2. Comparison of caesarean scar defects and symptoms at minimum 6-months follow-up postdelivery according to allocated closure type

$\left({ }^{1}=\right.$ Transvaginal ultrasonography ${ }^{2}=$ Saline infusion sonohysterography $)$

\section{Figure legends}

Figure 1. CONSORT flow diagram

Figure 2. Saline infusion sonohysterography evaluation of a patient's cesarean scar defects $\left({ }^{1}\right.$ length of niche, ${ }^{2}$ depth of niche, ${ }^{3}$ residual myometrium thickness, ${ }^{4}$ adjacent myometrium thickness, ${ }^{5}$ distance between niche and external os)

Figure 3. Saline infusion sonohysterography evaluation of a patient's cesarean scar defects ( ${ }^{1}$ width of niche)

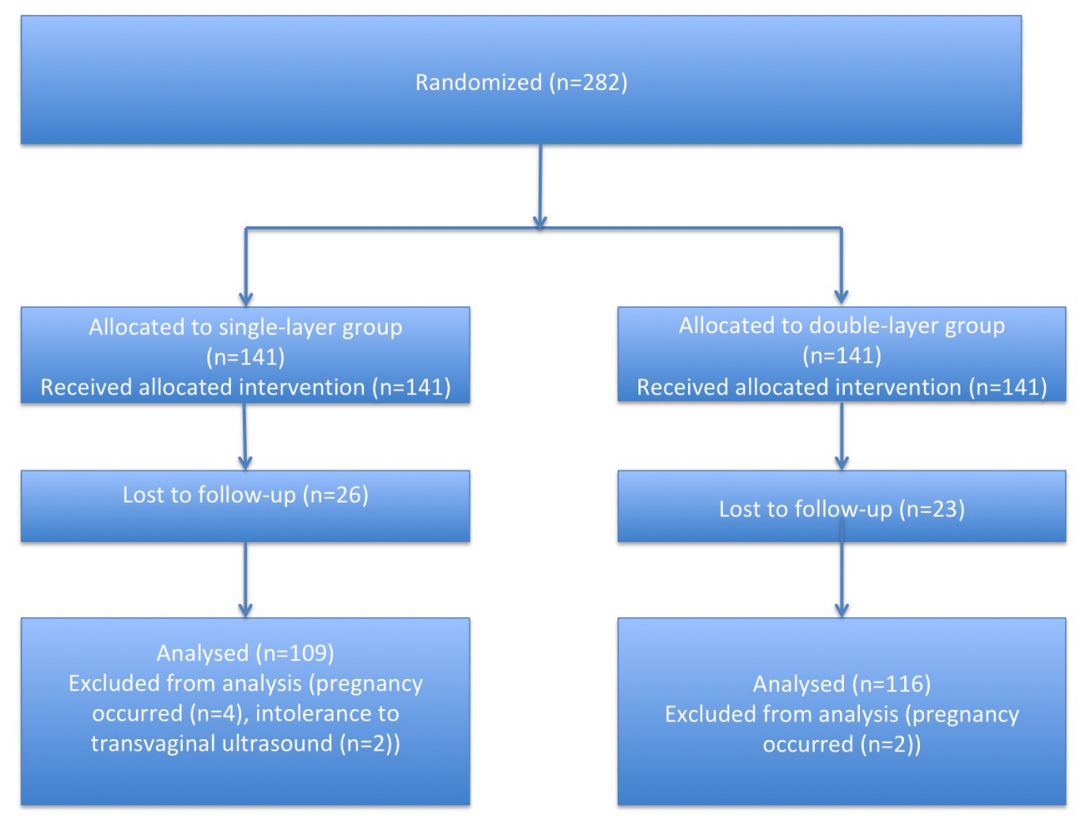



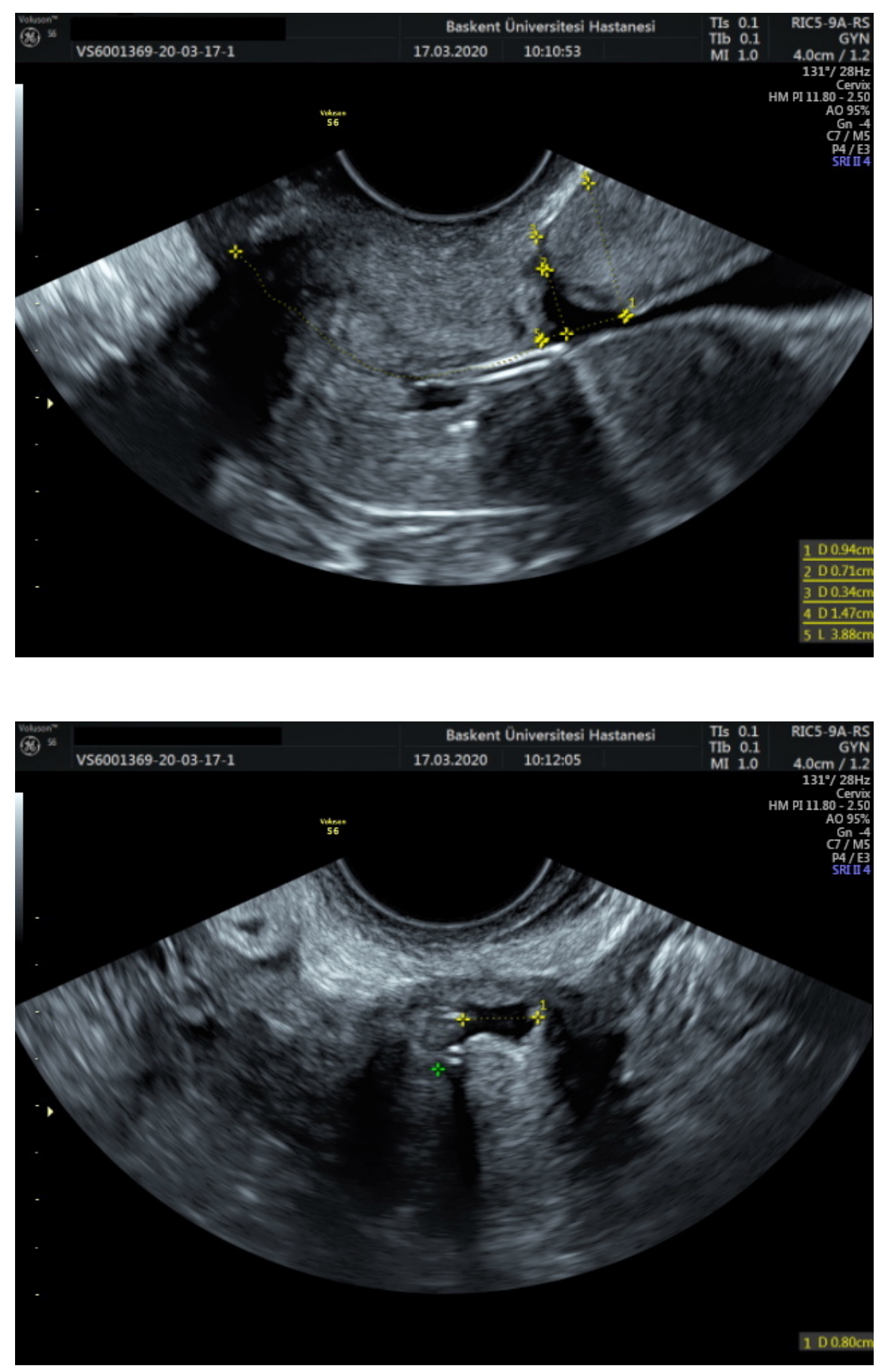